\title{
Bilateral coronary artery to pulmonary trunk fistulae detected by coronary angiography and evaluated by ECG-gated SPECT myocardial perfusion imaging: report of two cases
}

\author{
M. Pachowicz'1, 2, J. Drozd ${ }^{3}$, M. Bełz ${ }^{4}$, R. Maciejewski², B. Chrapko \\ ${ }^{1}$ Chair and Department of Nuclear Medicine, Medical University of Lublin, Poland \\ ${ }^{2}$ Chair and Department of Human Anatomy, Medical University of Lublin, Poland \\ ${ }^{3}$ Department of Invasive Cardiology, Home Office and Administration Hospital, Lublin, Poland \\ ${ }^{4}$ Chair and Department of Cardiology, Medical University of Lublin, Poland
}

[Received 20 January 2014; Accepted 23 March 2014]

Coronary artery fistulae (CAF) are anomalies related to coronary artery abnormal termination. This is a very rare congenital malformation accounting for about $0.2-0.4 \%$ of congenital cardiac anomalies, but in some patients it can be haemodynamically important.

Single-photon emission computed tomography or positron emission tomography myocardial perfusion imaging (MPI) using radioactive agents is widely used in clinical practice for cardiac ischaemia detection as a very sensitive and non-invasive tool. We are going to present 2 patients with bilateral CAFs to the pulmonary trunk without signs of the rest or stress ischaemia in MPI. (Folia Morphol 2014; 73, 4: 514-520)

Key words: coronary artery fistula, myocardial perfusion imaging, SPECT, myocardial ischaemia, computed tomography

\section{INTRODUCTION}

Coronary artery fistulae (CAF) are anomalous connections bypassing myocardial capillary network. It can be located between a coronary artery or one of its branches and the cardiac chamber lumen (coronary-cameral fistulae) or an extracardiac blood vessel, most commonly one of the great vessels: pulmonary trunk, pulmonary vein or superior vena cava (coronary-vascular fistulae) [4].

The coronary arteriovenous fistulae affect $0.002 \%$ of general population. The prevalence of this anomaly varies in literature, it is estimated that up to $0.4 \%$ of all congenital anomalies of the heart are CAF which are detected in up to $0.8 \%$ of patients undergoing coronary angiography $[1,3,4,16]$.
The fistulae are most commonly congenital, but can also be acquired. Most of them are small and asymptomatic [4, 7].

Radionuclide myocardial perfusion imaging (MPI) is a widely used modality in cardiac ischaemia evaluation and its importance is still growing despite alternative diagnostic tool development such as computed tomography (CT) or magnetic resonance imaging. The number of studies performed has significantly increased in the last decade [6].

MPI can be performed either by single photon emission tomography (SPECT) or positron emission tomography techniques utilising radioactive agents which accumulate in the myocardium reflecting myocardial blood flow. These methods are non-invasive, 
safe, possess high diagnostic value and enable prediction of the adverse cardiac events [10].

\section{STUDY PROTOCOL FOR MPI}

MPI was performed at the nuclear medicine department. In both cases the 2 day protocol was used with a 48-h delay between rest and stress studies.

A stress test was performed using a cycloergonometer. The initial load was 25 watts and was increased by 25 watts every 3 min until the submaximal heart rate was achieved under constant electrocardiography (ECG) monitoring and cardiologist supervision. Then the radiotracer was given intravenously and physical exertion was continued for $1 \mathrm{~min}$.

Both the rest and stress ECG-gated SPECT studies were done $60 \mathrm{~min}$ after intravenous injection of $740 \mathrm{MBq}$

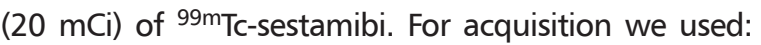
in patient 1 - gamma camera Varicam (Elscint, Haifa, Israel), in patient 2 - Symbia T16 SPECT/CT hybrid gamma camera (Siemens, Erlangen, Germany) with additional low-energy $C T$ study to provide the attenuation correction. The acquisition was performed with 2 gamma cameras in orthogonal configuration, in 32 views, $25 \mathrm{~s}$ for each with head movement of 3 degrees between views.

The studies were evaluated using dedicated workstations including both visual examination and semi-quantitative analysis using the following software: in patient 1 - Cedars-Sinai, in patient 2 - Invia Corridor 4DM.

\section{CASES PRESTENTATION}

\section{Patient 1}

Fifty-year-old male, with the history of acute coronary syndrome and percutaneous coronary intervention on the origin of his right coronary artery (RCA) with bare metal stent implantation, while staying abroad visited a local cardiologist due to the typical symptoms of the myocardial ischaemia. The stress test performed on a treadmill using Bruce protocol revealed multiple-lead ST segment depressions, so a coronary angiography was scheduled and carried out. The angiography showed "de novo" stenosis in the middle segment of the RCA (Fig. 1) as well as an abnormal vessel arising from its proximal segment and communicating with the pulmonary trunk (Fig. 2). Moreover, a second malformation was discovered in the proximal segment of the left anterior descending artery (LAD). It was a complex anomaly including a fistula draining to the pulmonary trunk. The mild stenosis of the LAD (branch of left coronary artery) at the origin of the first diagonal artery was also present (Figs. 3, 4).

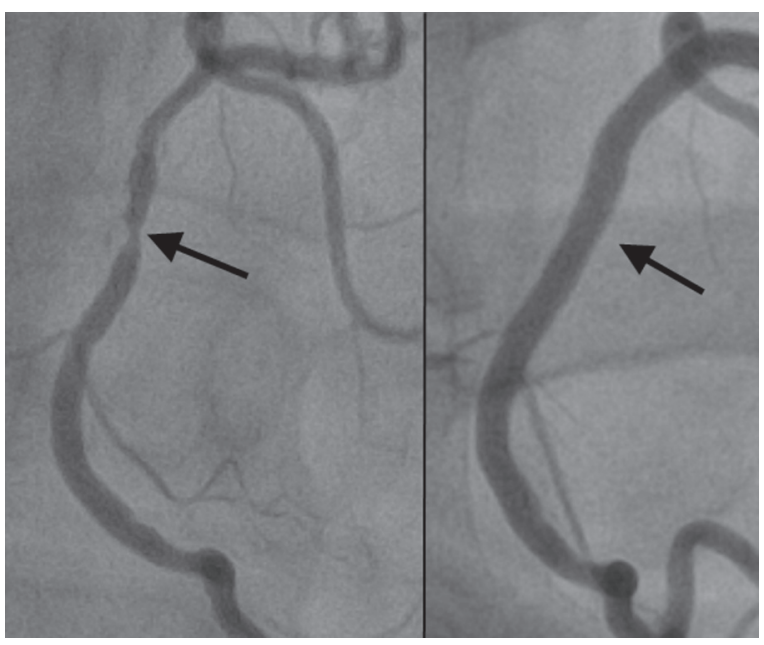

Figure 1. Patient 1. Right coronary artery before and after successful angioplasty (arrows).

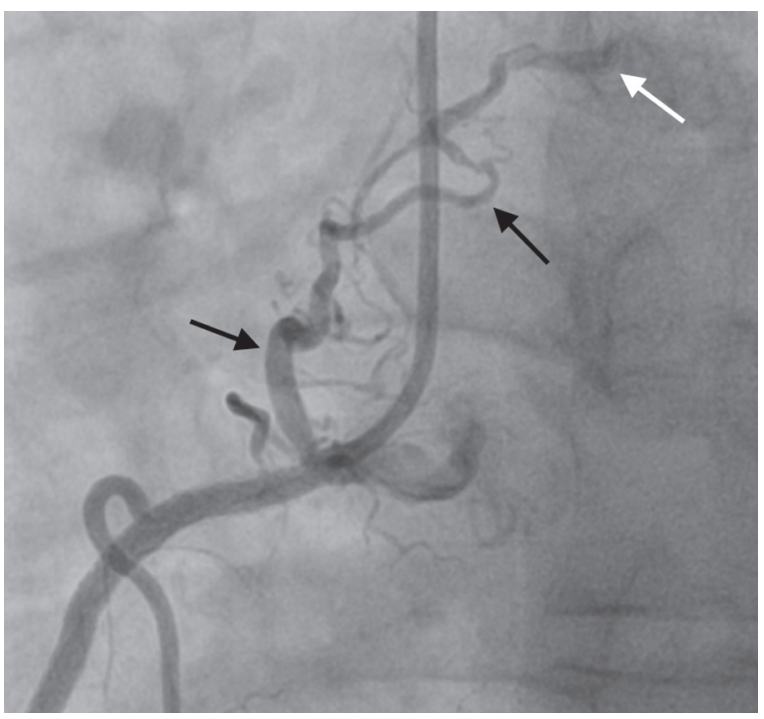

Figure 2. Patient 1. Right coronary artery. Coronaropulmonary fistula (black arrows). Pulmonary trunk (white arrow).

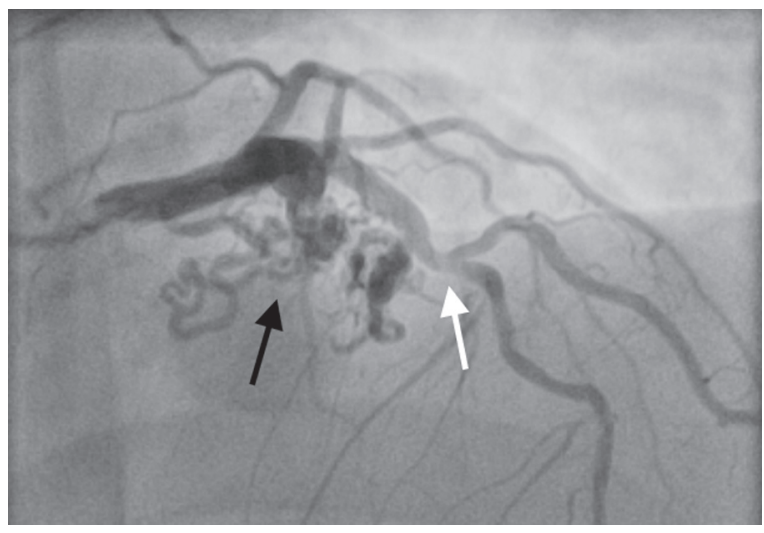

Figure 3. Patient 1. Left coronary artery. Mild stenosis in the point of origin of the first diagonal branch (white arrow). Blood vessels malformation (black arrow). 


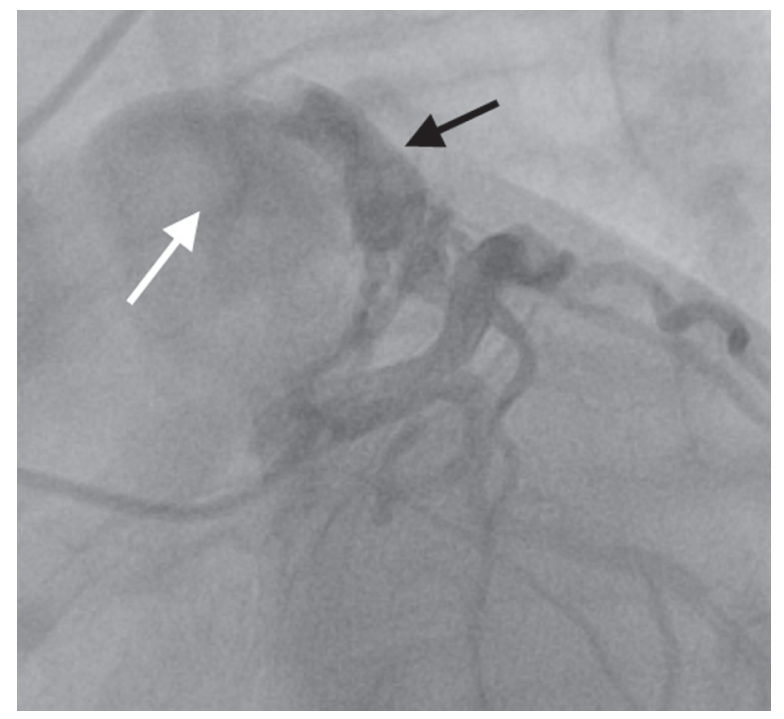

Figure 4. Patient 1. Left coronary artery. Coronaropulmonary fistula (black arrow). Pulmonary trunk (white arrow).

The angiography was followed by a successful coronary angioplasty of the RCA with the drug eluting stent implantation.

MPI was recommended for further evaluation of the anomalies' possible impact on the patient's condition. The study protocol described above was used. During the stress test sestamibi was injected intravenously at the heart rate $162 \mathrm{bpm}$ (90\% of maximal heart rate calculated for the patient). No features of cardiac ischaemia were present in the ECG during the physical exertion, which was well tolerated. No chest pain or other symptoms of ischaemia were reported by the patient. Both rest and stress studies revealed no significant ischaemia. Rest motion and thickening were normal and left ventricular ejection fraction (LVEF) was $58 \%$. After the stress test the motion and thickening of the interventricular septum were slightly worse and LVEF was $54 \%$ (Figs. 5, 6).

The patient remained asymptomatic and in good general condition during the control visit. The ECG was normal. A control echocardiography was performed showing normal left ventricular end-diastolic dimension (LVEDD $53 \mathrm{~mm}$ ), with good global contractility (LVEF 61\%) and hypokinetic apical part of the septum, slight enlargement of the left atrium (40 $\mathrm{mm})$, normal pulmonary trunk $(22 \mathrm{~mm}$ ) and right ventricle $(26 \mathrm{~mm})$, and minor mitral and tricuspid insufficiency.

\section{Patient 2}

Sixty-eight-year-old female with hypertension, diabetes, hypothyroidism, chronic obstructive pulmonary disease, history of transient ischaemic attack and paroxysmal atrial fibrillation was referred for coronary angiography due to typical symptoms of

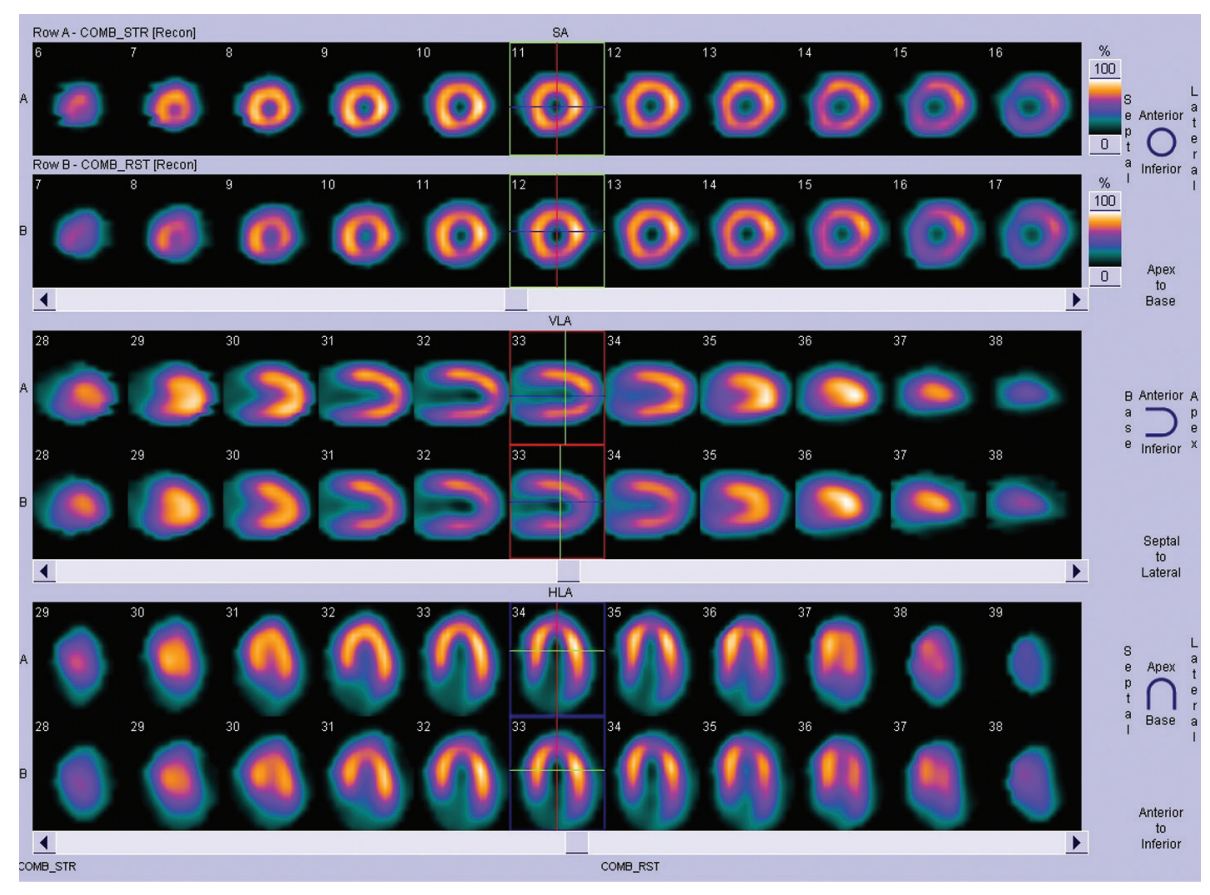

Figure 5. Patient 1. Normal myocardial stress-rest perfusion study. 


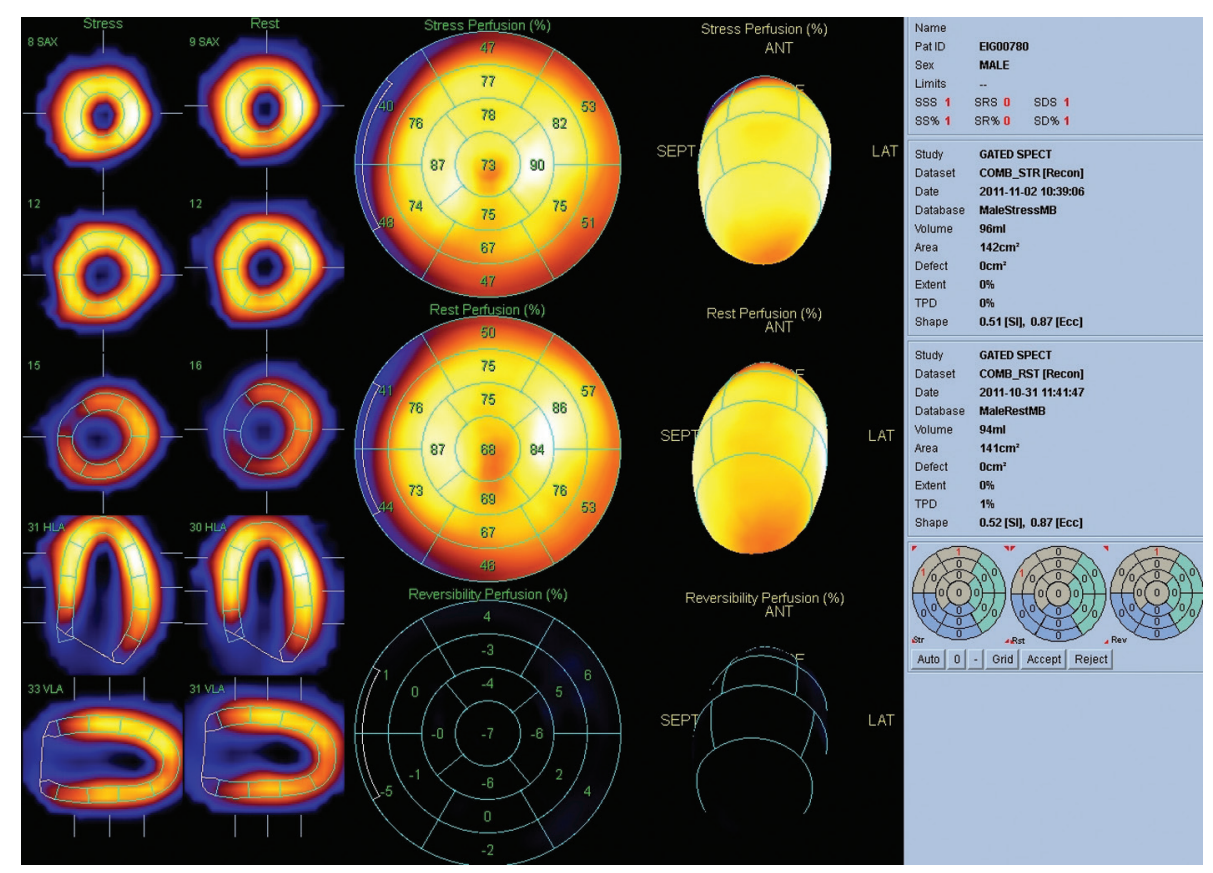

Figure 6. Patient 1. Semiquantification of myocardial stress-rest perfusion study — no significant ischaemia.

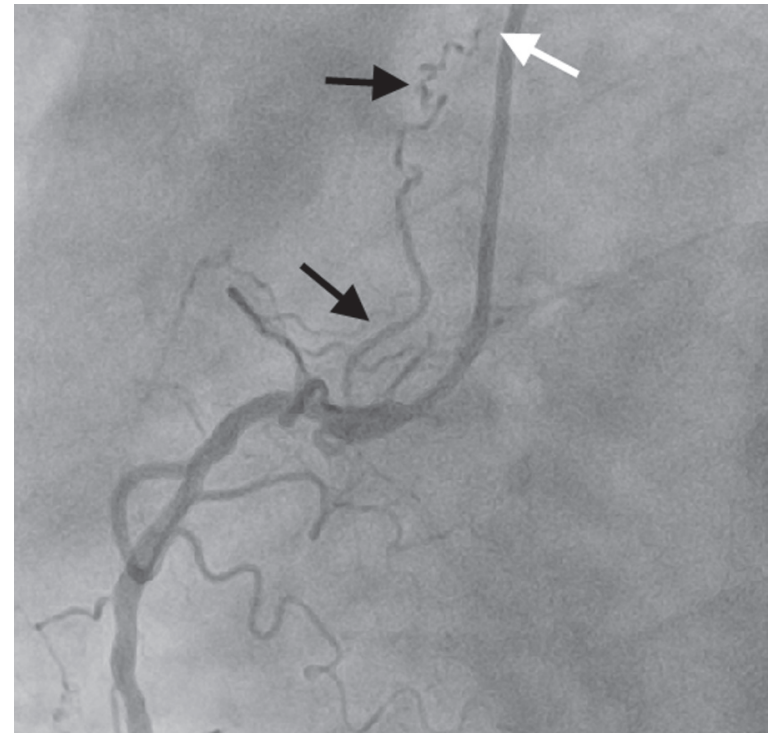

Figure 7. Patient 2. Right coronary artery. A fistula (black arrows) connecting right coronary artery to the pulmonary trunk (white arrow).

angina and a positive ECG stress test. In rest ECG inversion of the $T$ waves in leads $\mathrm{V}_{1}-\mathrm{V}_{3}$ was observed. Echocardiography showed normal LVEDD (49 $\mathrm{mm})$, with good global contractility (LVEF 65\%), slight enlargement of the right ventricle $(30 \mathrm{~mm})$, normal pulmonary trunk dimension $(23 \mathrm{~mm}$ ) and elevated pulmonary pressure estimated on tricuspid regurgitation peak gradient $(37 \mathrm{~mm} \mathrm{Hg})$.

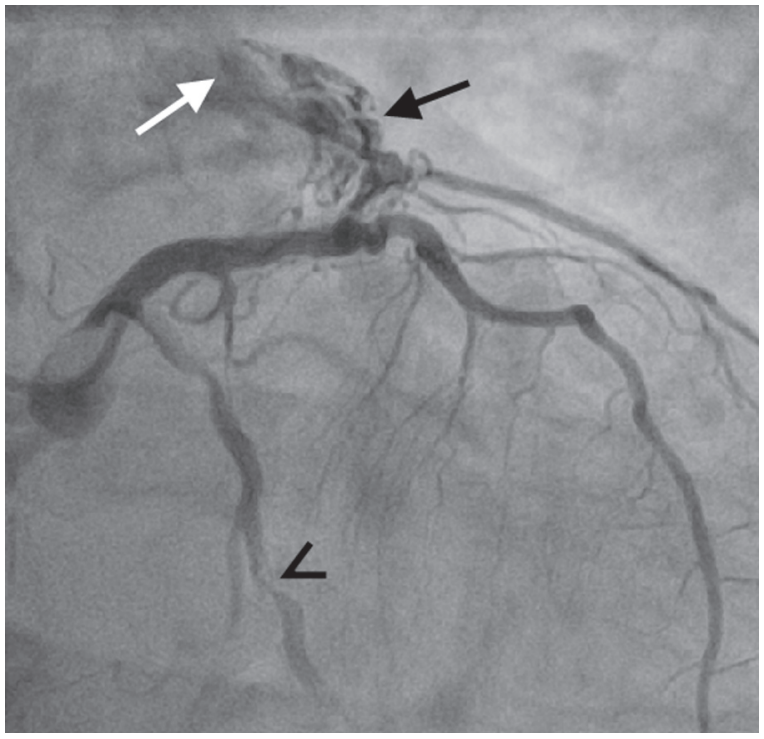

Figure 8. Patient 2. Left coronary artery. A plexiform like anomalous connection (black arrow) between the left anterior descending artery and pulmonary trunk (white arrow). Critical stenosis of the second obtuse marginal branch (arrowhead).

The study revealed critical stenosis of the large second obtuse marginal branch of the left coronary artery and borderline stenosis of the origin of the first marginal branch. Additionally, 2 malformations were found: a plexiform anastomosis originating from the right coronary sinus draining to the pulmonary trunk (Fig. 7) and a similar anastomosis between the middle part of the LAD and the pulmonary trunk (Fig. 8). 


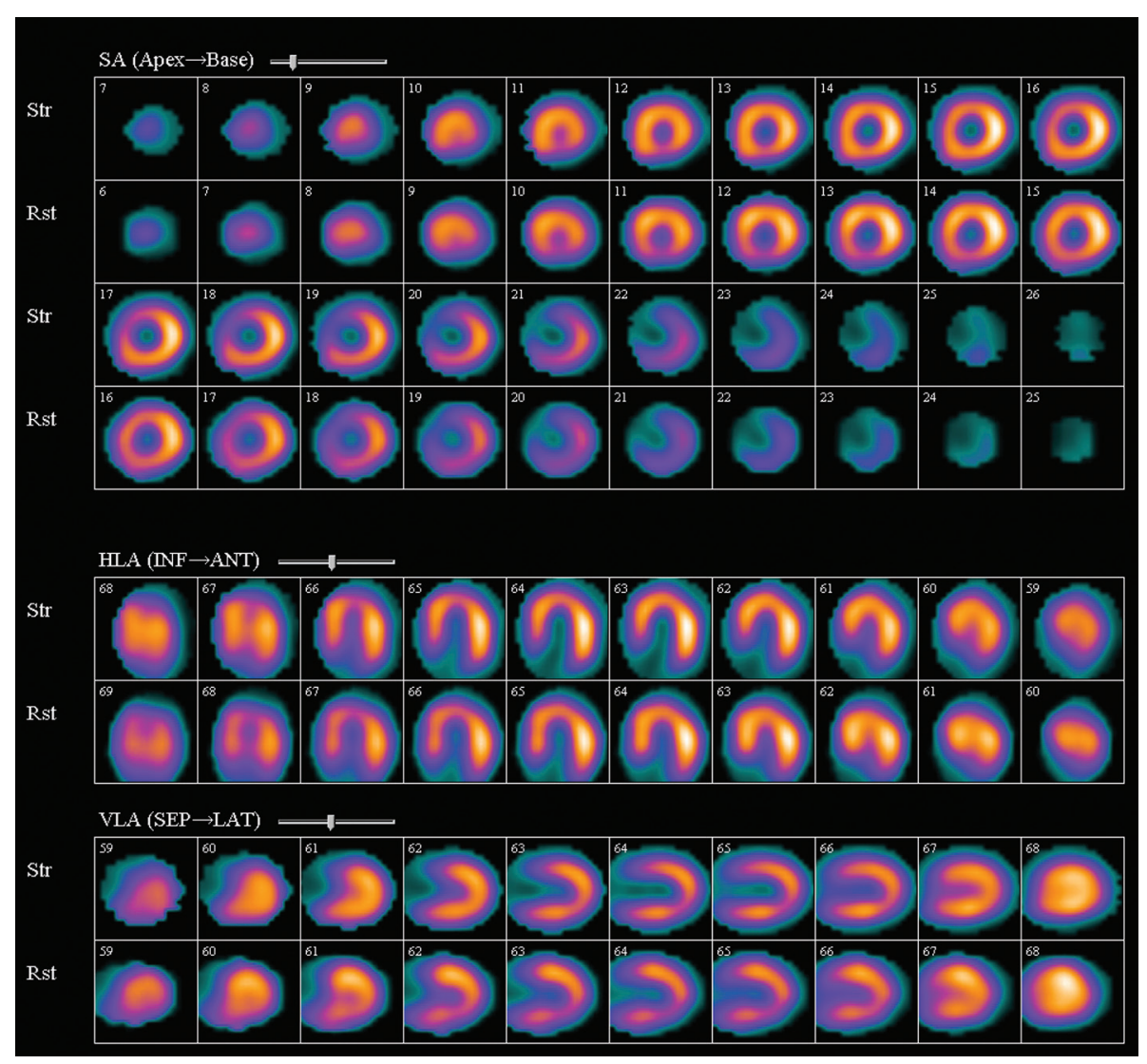

Figure 9. Patient 2. Stress-rest perfusion slice display, no attenuation correction images. No features of ischaemia.

During the stress test a radiotracer was injected at the heart rate of $120 \mathrm{bpm}$ ( $80 \%$ of maximal heart rate calculated for the patient). Physical exertion was poorly tolerated nonetheless ECG presented no signs of ischaemia. Heart rate increased rapidly and the patient was quickly fatigued, but she did not report any distressing symptoms.

The rest study revealed no significant ischaemia both in no-attenuation correction and attenuation correction images, only mild ischaemia of the apical lateral segment was suggested after detailed evaluation. No abnormal motion or thickening of the left ventricle muscle was found with LVEF $77 \%$. Stress study presented no significant differences compared to rest study. LVEF was 79\% (Figs. 9, 10).

Based on the symptoms and the stress studies the decision of the percutaneous coronary intervention was taken. The uneventful intervention on the marginal branch with a bare metal stent implantation was performed. After this procedure the patient remained asymptomatic and in good general condition.

\section{DISCUSSION}

Most frequently, the CAF originates from the RCA, less commonly - from the LAD. The circumflex branch of the left coronary artery is rarely affected [4]. In some studies though, the proportions were different. Said's et al. [13] reported LAD as the most common point of origin for CAF. In the same study, the unilateral fistula was reported in $80 \%$, bilateral in $18 \%$ and multilateral in $2 \%$ of patients.

The point of termination for CAFs is most commonly the pulmonary trunk, less frequently the right-sided heart chambers, left atrium, superior vena cava or coronary sinus, rarely the left ventricle $[12,13]$.

Large CAFs may lead to the steal phenomenon resulting in reduced blood flow in the area of arterial supply [7]. The size of such a connection, when a fistula becomes haemodynamically significant and signs and symptoms develop, is about $200 \%$ of the coronary vessel. Thus, symptoms and complications are less common in young patients, but the risk increases with age as the fistula enlarges. This may eventually lead to severe complications: myocardial ischaemia sudden cardiac death, endocarditis or 


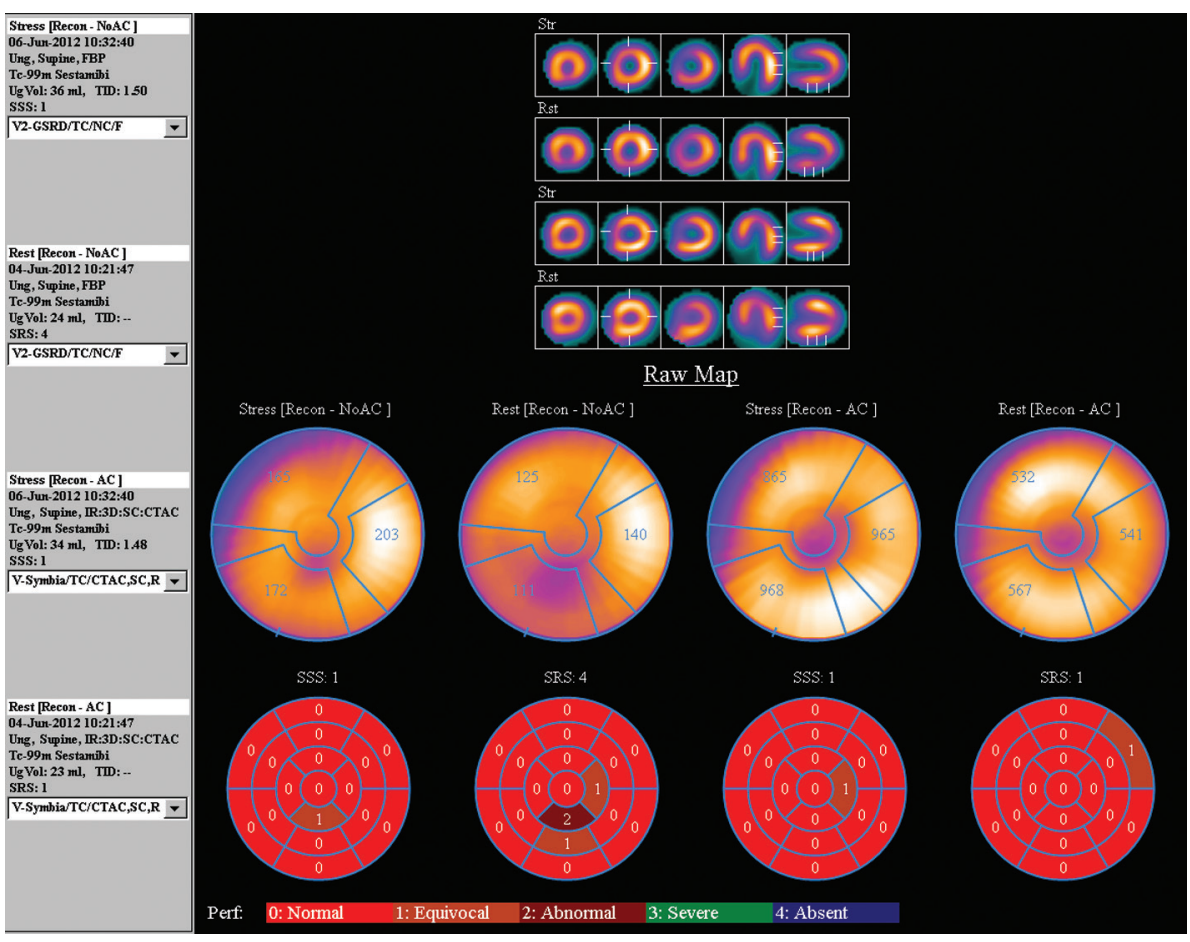

Figure 10. Patient 2. Semiquantification of myocardial stress-rest perfusion study — no significant ischaemia.

secondary aortic and mitral valves dysfunctions $[1,4,13]$.

The CAF is challenging as many patients remain asymptomatic or their symptoms mimic different heart diseases. The patient may complain of dyspnoea, fatigue or angina. The physical examination may reveal different murmurs due to the fistula itself or secondary to valvular dysfunction, alterations in pulse or even congestive heart failure signs [4, 13].

The coronary angiography is a gold standard in detection of the CAFs and usually such anomalies are revealed accidentally [4]. Still, the coronary angiography has some limitations and is an invasive procedure. The CT coronary angiography is a non-invasive modality and enables 3-dimensional observation of the blood vessels as well as simultaneous evaluation of the cardiac function [5, 9, 12, 13].

Radionuclide modalities have also been used in the evaluation of patients with CAFs, but available information is mainly limited to single cases. Rubini et al. [11] evaluated 9 patients with known CAFs. MPI with ${ }^{99 m}$ Tc-sestamibi detected ischaemic changes in areas corresponding to coronary arteries with fistulae only in 2 patients. There are multiple cases reported with different types of CAFs who underwent MPI. Obtained results range from normal stress and rest perfusion to significant ischaemia in different patients $[2,8,14,15]$.
Still only a small number of patients with CAF suffer from cardiac ischaemia related to this anomaly [11].

\section{CONCLUSIONS}

The CAF require a multidisciplinary approach and an evaluation may be completed by cooperation of the cardiologist, radiologist and nuclear medicine physician.

In the presented cases no interventions were taken on the coronary fistulae as there were normal MPI. Since both patients remain asymptomatic and in good condition, the treatment strategy based on radionuclide method seems accurate. This strongly suggests that information provided by MPI may enable proper selection of patients requiring invasive treatment and avoid unnecessary risk in those who do not need it.

\section{REFERENCES}

1. Ata Y, Turk T, Bicer M, Yalcin M, Ata F, Yavuz S (2009) Coronary arteriovenous fistulas in the adults: natural history and management strategies. J Cardiothorac Surg, 4: 62.

2. Barone-Rochette G, Vanzetto G, Saunier C, Machecourt J (2009) Combination of anatomic and perfusion imaging for decision making in a professional soccer player with giant coronary artery to left ventricle fistula. J Nucl Cardiol, 16: 640-643.

3. Canga Y, Ozcan KS, Emre A, Kul S, Guvenc TS, Durmus G, Kirbas V, Ilhan E, Karatas MB, Oz D, Terzi S, Yesilcimen $K$ 
(2012) Coronary artery fistula: review of 54 cases from single center experience. Cardiol J, 19: 278-286.

4. Majidi M, Shahzamani M, Mirhoseini M (2011) Clinical features of coronary artery fistula. J Tehran Heart Cent, 6: 158-162.

5. Natarajan A, Khokhar AA, Kirk P, Patel HH, Turner D (2013) Coronary-pulmonary artery fistula: value of 64-MDCT imaging. QJM, 106: 91-92.

6. Notghi A, Low CS (2011) Myocardial perfusion scintigraphy: past, present and future. Br J Radiol, 84: S229-S236.

7. Oh JH, Lee HW, Cha KS (2012) Hemodynamic significance of coronary cameral fistula assessed by fractional flow reserve. Korean Circ J, 42: 845-848.

8. Palloshi A, Aprigliano G (2008) Myocardial ischemia due to a large coronary-pulmonary fistula with plexus-like morphology. J Cardiovasc Med (Hagerstown), 9: 1163-1165.

9. Pannu HK, Flohr TG, Corl FM, Fishman EK (2003) Current concepts in multidetector row CT evaluation of coronary arteries: principles, techniques and anatomy. Radiographics, 23: S111-S125.

10. Parker MW, Iskandar A, Limone B, Perugini A, Kim H, Jones $C$, Calamari B, Coleman Cl, Heller GV (2012) Diagnostic accuracy of cardiac positron emission tomography versus single photon emission computed tomography for coronary artery disease: a bivariate meta-analysis. Circ Cardiovasc Imag, 5: 700-707.

11. Rubini G, Ettorre GC, Sebastiani M, Bovenzi F (2000) Evaluation of hemodynamic significance of arteriovenous coronary fistulas: diagnostic integration of coronary angiography and stress/rest myocardial scintigraphy. Radiol Med, 100: 453-458.

12. Said SA (2010) Congenital solitary coronary artery fistulas characterized by their drainage sites. World J Cardiol, 26: 6-12.

13. Said SA (2011) Current characteristics of congenital coronary artery fistulas in adults: a decade of global experience. World J Cardiol, 3: 267-277.

14. Suchon E, Kostkiewicz M, Szot W (2012) Left coronary arteriovenous malformation with fistulous connections to the left and right ventricles. Nucl Med Rev Cent East Eur, 15: 80-82.

15. Vijayvergiya R, Bhadauria PS, Jeevan $H$, Mittal BR, Grover A (2010) Myocardial ischemia secondary to dual coronary artery fistulas draining into main pulmonary artery. Int J Cardiol, 140: e30-e33.

16. Yun H, Zeng MS, Yang S, Jin H, Yang X (2011) Congenital coronary artery fistulas: dual-source CT findings from consecutive 6624 patients with suspected or confirmed coronary artery disease. Chin Med J (Engl), 124: 4172-4177. 